\title{
Chapter
}

Anaesthesia, Pain, Intensive Care and Emergency Medicine - A.P.I.C.E.

pp 217-223

\section{Antioxidant Activity in the Therapy of Sepsis: From Experimental Data to Clinical Practice}

- G. P. Novelli

- , R. Casali

- , S. Falsini

- , E. Pieraccioli

\section{Abstract}

An antioxidant is defined as any substance which delays or prevents the oxidation of a substrate when it is present in small amounts relative to the amount of the substrate itself. Antioxidants are active at several levels of the oxidative sequence and their mechanism of action may be unique.

\section{References}

1. Lefer AM, Araki H, Okamatsu S (1981) Beneficial actions of a free radical scavenger in traumatic shock and myocardial ischemia. Circ Shock 8:273-282

PubMed (http://www.ncbi.nlm.nih.gov/entrez/query.fcgi?cmd=Retrieve\&db=PubMed\&dopt=Abstract\&list_uids=7249259)

2. Novelli GP, De Gaudio AR (1983) Oxygen free-radicals in shock states. Elsevier, Amsterdam

3. De Gaudio AR, Sarti A, Palmarini M et al (1983) Prevenzione dello shock sperimentale mediante uno scavenger di radicali liberi: il glutatione ridotto. Acta Anaesth Ital 34:501-507

4. Saez JC, Ward PH, Gunther B et al (1984) Superoxide radical involvement in the pathogenesis of burn shock. Circ Shock 12:229-233 PubMed (http://www.ncbi.nlm.nih.gov/entrez/query.fcgi?cmd=Retrieve\&db=PubMed\&dopt=Abstract\&list_uids=6327114)

5. Novelh GP (1992) Oxygen radicals in experimental shock: effects of spin-trapping nitrones in ameliorating shock pathophysiology. Crit Care Med 20:499-507

CrossRef (http://dx.doi.org/10.1097/00003246-199204000-00012)

6. Novelh GP, Angiolini P, Tani R et al (1985) Phenyl-t-butyl-nitrone is active against traumatic shock in rats. Free Radic Res Commun $1: 321-327$

CrossRef (http://dx.doi.org/10.3109/10715768609080971)

7. Goode HF, Webster NR (1993) Free radicals and antioxidants in sepsis. Crit Care Med 21: 1770-1776

PubMed (http://www.ncbi.nlm.nih.gov/entrez/query.fcgi?cmd=Retrieve\&db=PubMed\&dopt=Abstract\&list_uids=8222696) CrossRef (http://dx.doi.org/10.1097/00003246-199311000-00029)

8. Schiller HJ, Reilly PM, Bulkley GB (1993) Antioxidant therapy. Crit Care Med 21:S92-102

PubMed (http://www.ncbi.nlm.nih.gov/entrez/query.fcgi?cmd=Retrieve\&db=PubMed\&dopt=Abstract\&list_uids=8428505) CrossRef (http://dx.doi.org/10.1097/00003246-199302001-00016)

9. Rice-Evans CA, Diplock AT (1993) Current status of antioxidant therapy. Free Radic Biol Med 15:77-96

PubMed (http://www.ncbi.nlm.nih.gov/entrez/query.fcgi?cmd=Retrieve\&db=PubMed\&dopt=Abstract\&list_uids=8359712) $\underline{\text { CrossRef }}$ 
(http://dx.doi.org/10.1016/o891-5849(93)90127-G)

10. Bone RC (1992) Inhibitors of complement and neutrophils: a critical evaluation of their role in the treatment of sepsis. Crit Care Med 20:891-898

$\underline{\text { PubMed (http://www.ncbi.nlm.nih.gov/entrez/query.fcgi?cmd=Retrieve\&db=PubMed\&dopt=Abstract\&list_uids=1597046) CrossRef }}$ (http://dx.doi.org/10.1097/00003246-199206000-00029)

11. Youn YK, Lalonde C, Demling R (1991) Use of antioxidant therapy in shock and trauma. Circ Shock 35:245-249

PubMed (http://www.ncbi.nlm.nih.gov/entrez/query.fcgi?cmd=Retrieve\&db=PubMed\&dopt=Abstract\&list_uids=1777961)

12. Mannion D, Fitzpatrick GJ, Feeley M (1994) Role of xanthine oxidase inhibition in survival from hemorrhagic shock. Circ Shock 42:3943

PubMed (http://www.ncbi.nlm.nih.gov/entrez/query.fcgi?cmd=Retrieve\&db=PubMed\&dopt=Abstract\&list_uids=8149508)

13. Sahm AS (1991) Protection against stress-induced acute gastric mucosal injury by free-radical scavengers. Intensive Care Med 17:455460

CrossRef (http://dx.doi.org/10.1007/BF01690766)

14. Tani T, Aoki H, Yoshioka T (1993) Treatment of septic shock with a protease inhibitor in a canine model: a prospective, randomized, controlled trial. Crit Care Med 21:925-930

PubMed (http://www.ncbi.nlm.nih.gov/entrez/query.fcgi?cmd=Retrieve\&db=PubMed\&dopt=Abstract\&list_uids=8389269) $\underline{\text { CrossRef }}$ (http://dx.doi.org/10.1097/00003246-199306000-00023)

15. Novelli GP, Innocenti P, Livi P (1993) II gabesato mesilato (Foy) nuovo antiproteasico sintetico nel trattamento dello shock. Studio multicentrico italiano. Minerva Anestesiol 58: 247-253

16. Novelh GP, Casali R, Bonizzoh M et al (1995) Azione antiossidante del gabesato mesilato (Foy) in un modello sperimentale di shock. Minerva Anestesiol 60 (in press)

17. Sanfey H, Bulkley GB, Cameron JL (1985) The pathogenesis of acute pancreatitis. The source and the role of oxygen-derived free radicals in three different experimental models. Ann Surg 201:633-639

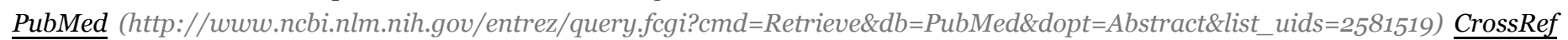
(http://dx.doi.org/10.1097/00000658-198505000-00013)

18. Fujishima S, Aikawa N (1995) Neutrophil-mediated tissue injury and its modulation. Intensive Care Med 21:277-285

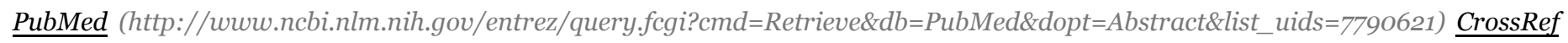
(http://dx.doi.org/10.1007/BFo1701489)

19. Wolbarsht ML, Fridovich I (1989) Hyperoxia during reperfusion is a factor in reperfusion injury. Free Radic Biol Med 6:61-62 PubMed (http://www.ncbi.nlm.nih.gov/entrez/query.fcgi?cmd=Retrieve\&db=PubMed\&dopt=Abstract\&list_uids=2536344) CrossRef (http://dx.doi.org/10.1016/0891-5849(89)90161-5)

20. Drugas GT, Paidas CN, Yahanda AM et al (1991) Conjugated desferoxamine attenuates hepatic microvascular injury following ischemia-reperfusion. Circ Shock 34:278-283

PubMed (http://www.ncbi.nlm.nih.gov/entrez/query.fcgi?cmd=Retrieve\&db=PubMed\&dopt=Abstract\&list_uids=1934329)

21. Fleckenstein AE, Smith SL, Linseman KL et al (1991) Comparison of the efficacy of mechanistically different antioxidants in the rat hemorrhagic shock model. Circ Shock 35: 223-230

PubMed (http://www.ncbi.nlm.nih.gov/entrez/query.fcgi?cmd=Retrieve\&db=PubMed\&dopt=Abstract\&list_uids=1777958)

22. Zhao W, Richardson JS, Mombourquette MJ et al (1995) An in vitro EPR study of the free radical scavenging actions of the lazaroid antioxidants U-74500 A and U-78517 F. Free Radic Biol Med 19:21-30

PubMed (http://www.ncbi.nlm.nih.gov/entrez/query.fcgi?cmd=Retrieve\&db=PubMed\&dopt=Abstract\&list_uids=7635355) CrossRef (http://dx.doi.org/10.1016/0891-5849(95)00007-K)

23. Johnson G, Lefer AM (1990) Protective effects of a novel 21-aminosteroid during splanchnic artery occlusion shock. Circ Shock 30:155-164

PubMed (http://www.ncbi.nlm.nih.gov/entrez/query.fcgi?cmd=Retrieve\&db=PubMed\&dopt=Abstract\&list_uids=2311204)

24. Inove M, Saito Y, Hirata E et al (1987) Regulation of redox states of plasma proteins by metabolism and transport of glutathione and related compounds. J Prot Chem 6:207-225

25. Meister A (1988) Glutathione metabohsm and its selective modification. J Biol Chem 263: 17205-17208

PubMed (http://www.ncbi.nlm.nih.gov/entrez/query.fcgi?cmd=Retrieve\&db=PubMed\&dopt=Abstract\&list_uids=3053703)

26. Robinson MK, Ahn MS, Rounds JD (1992) Parenteral glutathione monoester enhaucas tissue antioxidant stores. JPEN J Parenter Enteral Nutr 16:413-418

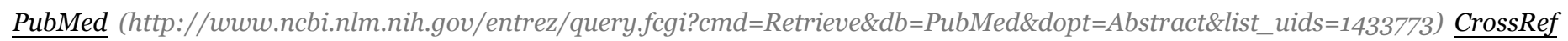
(http://dx.doi.org/10.1177/0148607192016005413)

27. Griffith OW, Meister A (1985) Origin and turnover of mitochondrial glutathione. Proc Natl Acad Sci USA 82:4668-4672 $\underline{\text { PubMed (http://www.ncbi.nlm.nih.gov/entrez/query.fcgi?cmd=Retrieve\&db=PubMed\&dopt=Abstract\&list_uids=3860816) CrossRef }}$ (http://dx.doi.org/10.1073/pnas.82.14.4668)

28. Hagen TM, Yee AWT, Jones DP (1988) Glutathione uptake and protection against oxidative injury in isolated kidney cells. Kidney Int 
PubMed (http://www.ncbi.nlm.nih.gov/entrez/query.fcgi?cmd=Retrieve\&db=PubMed\&dopt=Abstract\&list_uids=3172638) $\underline{\text { CrossRef }}$ (http://dx.doi.org/10.1038/ki.1988.147)

29. Falsini S, Celiai MP, Angiolini P et al (1994) Glutatione ridotto e L-cisteina nello shock endotossinico nel ratto. Minerva Anestesiol 60:413-418

PubMed (http://www.ncbi.nlm.nih.gov/entrez/query.fcgi?cmd=Retrieve\&db=PubMed\&dopt=Abstract\&list_uids=7808645)

30. Novelli GP, Falsini S, Bracciotti G (1991) Exogenous glutathione increases endurance to muscle effort in mice. Pharmacol Res 23:149155

PubMed (http://www.ncbi.nlm.nih.gov/entrez/query.fcgi?cmd=Retrieve\&db=PubMed\&dopt=Abstract\&list_uids=206279o) $\underline{\text { CrossRef }}$ (http://dx.doi.org/10.1016/S1043-6618(05)80116-1)

31. Scardi S, Rossi R, Pieraccioli E et al (1993) Reduced glutathione prevents lipid peroxidation as expressed by ethane exhalation during aortic surgery in man. Minerva Anestesiol 59:S2-215

32. Mediati RD, Girardi G, Rossi R et al (1993) Glutathione administration prevents the increase of extravascular lung water during ischemia and reperfusion in man. Minerva Anestesiol 59: S2-214

33. Paternostro E, Scardi S, Pellegrini G (1993) Lung lipoperoxidative stress during hyperoxic anaesthesia: protective effects of reduced glutathione. Minerva Anestesiol 59:S2-213

34. Novelli GP, Casali R, Bonizzoli M et al (1993) Aumento della permeabilità capillare provocato dall'endotossina: protezione con antiossidanti e glutatione. Minerva Anestesiol 59: 211-216

PubMed (http://www.ncbi.nlm.nih.gov/entrez/query.fcgi?cmd=Retrieve\&db=PubMed\&dopt=Abstract\&list_uids=8355861)

35. Di Filippo A, Paternostro E, Scardi S et al (1992) Insufficienza multipla d'organo sperimentale nel ratto: effetti protettivi del glutatione ridotto. Acta Anaesth Ital 43:358-369

36. Novelli GP, Melani AM, Consales G et al (1994) Antioxidant drugs in cerebral and spinal ischemia. Minerva Anestesiol 60:543-546 PubMed (http://www.ncbi.nlm.nih.gov/entrez/query.fcgi?cmd=Retrieve\&db=PubMed\&dopt=Abstract\&list_uids=7830916)

37. Novelh GP (1990) I radicali liberi nello shock e in alcuni quadri ad esso correlati. In Albano et al (eds): Radicali liberi in patologia nuovi orientamenti patogenetici e strategie chniche. Prag 6-9 December 1990

38. Aruoma OI, Halliwell B, Hoey BM (1989) The antioxidant properties of n-acetyl-cysteine. Free Radic Biol Med 6:593-597

PubMed (http://www.ncbi.nlm.nih.gov/entrez/query.fcgi?cmd=Retrieve\&db=PubMed\&dopt=Abstract\&list_uids=2546864) CrossRef (http://dx.doi.org/10.1016/0891-5849(89)90066-X)

39. Bernard GR (1991) N-acetylcysteine in experimental and clinical acute lung injury. Am J Med 91:S3 54-59

CrossRef (http://dx.doi.org/10.1016/0002-9343(91)90284-5)

40. Spies CD, Reinahart K, Witt I et al (1994) Influence of n-acetylcysteine on indirect indicators of tissue oxygenation in septic shock patients: results from a prospective, randomized, double- blind study. Crit Care Med 22:1738-1746

PubMed (http://www.ncbi.nlm.nih.gov/entrez/query.fcgi?cmd=Retrieve\&db=PubMed\&dopt=Abstract\&list_uids=7956276)

41. Zimmerman JJ (1992) Radical viewpoints in critical illness. Crit Care Med 20:448-449

PubMed (http://www.ncbi.nlm.nih.gov/entrez/query.fcgi?cmd=Retrieve\&db=PubMed\&dopt=Abstract\&list_uids=1559355) CrossRef (http://dx.doi.org/10.1097/00003246-199207000-00003)

42. Yamauchi N, Watanabe N, Kuriyama H et al (1990) Suppressive effects of intracellular glutathione on hydroxyl radical production induced by tumor necrosis factor. Int J Cancer 46: 884-888

$\underline{\text { PubMed (http://www.ncbi.nlm.nih.gov/entrez/query.fcgi?cmd=Retrieve\&db=PubMed\&dopt=Abstract\&list_uids=2172172) CrossRef }}$ (http://dx.doi.org/10.1002/ijc.2910460522)

\section{About this Chapter}

Title

Antioxidant Activity in the Therapy of Sepsis: From Experimental Data to Clinical Practice Book Title

Anaesthesia, Pain, Intensive Care and Emergency Medicine - A.P.I.C.E.

Book Subtitle

Proceedings of the 10th Postgraduate Course in Critical Care Medicine Trieste, Italy — November 13-19, 1995

Pages

Copyright

pp 217-223

1996 
DOI

Print ISBN

10.1007/978-88-470-2203-4_17

Online ISBN

978-3-540-75014-7

978-88-470-2203-4

Publisher

Springer Milan

Copyright Holder

Springer-Verlag Italia, Milano

Additional Links

- $\underline{\text { About this Book }}$

Topics

- Intensive / Critical Care Medicine

- Anesthesiology

Industry Sectors

- Electronics

- Health \& Hospitals

- Biotechnology

- Pharma

eBook Packages

- $\underline{\text { Springer Book Archive }}$

Editors

- Prof. Antonino Gullo M.D. ${ }^{(1)}$

Editor Affiliations

- 1. Department of Anaesthesiology and Intensive Care, Trieste University School of Medicine

Authors

- G.P. Novelli

- $\frac{\text { R. Casali }}{\text { S. }}$

- S. Falsini

- $\underline{\text { E. Pieraccioli }}$ 